\title{
The effect of administrating hyoscine bromide on pregnancy rate before embryo transfer in ART cycles
}

\author{
Mahvash Zargar, Shiva Kajbaf, Masoud Hemadi* \\ Fertility, Infertilty and Perinatology Research Center, School of Medicine, Ahvaz Jundishapur University of Medical Sciences, Ah- \\ vaz, Iran \\ Email: ${ }^{*}$ mhemadi79@gmail.com
}

Received 27 May 2013; revised 26 June 2013; accepted 4 July 2013

Copyright (C) 2013 Mahvash Zargar et al. This is an open access article distributed under the Creative Commons Attribution License, which permits unrestricted use, distribution, and reproduction in any medium, provided the original work is properly cited.

\begin{abstract}
Assisted reproductive technology methods i.e., IVF and ICSI were extensively used for treatment of infertility. Although, these methods have many advantages but ART pregnancy rate remains low (30\% $35 \%)$. It seems that uterine peristalsis occurred after rhCG administration in turn resulting to impair implantation of the transferred embryos. Therefore this study was conducted to possess of whether hyoscine bromide can effectively alleviate uterine contraction and then have any effect on the reproductive outcome. Healthy women who underwent IVF or ICSI cycles (n = 142) were randomly assigned to one of two groups at the start of the cycle. Group control $(n=71)$ without applied hyoscine and group experiment $(n=71)$ applied hyoscine (25 mg). Controlled ovarian hyperstimulation was induced with follicle stimulating hormone (FSH). Sperm preparation and ovarian puncture for in vitro maturation and fertilization (IVF) of oocyte were carried out for 42 hours after rhCG injection. Two weeks after rhCG injection, bhCG pregnancy test to determine pregnancy outcome in both groups was done and the outcome results, along with other information were recorded separately. The pregnancy rate in experimental group was significantly higher than that of control group. when all of the cycles with or without giving $25 \mathrm{mg}$ of hyosine were stratified by the mean total follicles (more/less than 2 follicles) and embryos (more/less than $25 \mathrm{~kg} / \mathrm{m}^{2}$ ), the Patients' age, the quality grade of embryos and infertility duration (more/less than 5 years), the reproductive outcome, in overall, in the subjects with more than 10 oocytes, 5 embryos, more than quality $A$ and $B$ grades of embryos, less than 35 years age and also less than 5 years infertility duration was more pro-
\end{abstract}

${ }^{*}$ Corresponding author. nounced than the other patients. But the other parameter was not affected by the reproductive outcome. Clinical or statistical improvement of the reproductive outcome could be demonstrated in the hyosine treated group especially with increasing the mean total follicles, embryo, quality of embryo in women with low age and infertility duration. However, further well-designed studies are essential to offer a final conclusion.

Keywords: Assisted Reproductive Technology (ART); Hyoscine; Pregnancy; Embryo Transfer

\section{INTRODUCTION}

Assisted reproductive techniques (ART) include all the ways in which maturation and fertilization of the follicular oocyte by spermatozoa is done outside the uterine and in vitro $[1,2]$. IVF and embryo transfer techniques in the late 1970s and the technique of intracytoplasmic sperm injection (ICSI) in 1992 are presented [3].

Although these techniques are caused increasing the rate of clinical pregnancy, but nevertheless, they are faced with some constraints. Indeed, Most of these transfers are faced with the loss of the embryo before they take a chance to implant in the uterus [3,4].

It was suggested that stimulation of the ovaries prior to embryo transfer caused high level of estrogen which subsequently increases the intensity of uterine contractions, 1 as what was observed during menstruation [5]. The presence of uterine contractions and the imbalance hormone may be important factors that impede the successful transfer of embryos before embryo transfer, due to hormone therapy [6]. Therefore it is better to prevent the onset of uterine contractions during embryo transfer.

Real time-ultrasound scan analysis has shown that implantation and pregnancy rate decreases with increasing 
contractions of myometrium [2].

Several factors have been identified that they could cause prevention from these contractions. One of the most useful drugs that have been proposed to reduce uterine contractions is hyoscine bromide $[7,8]$.

Hyoscine bromide is one of the anticholinergic agents inhibiting acetylcholine receptors of the postganglionic nerves that can produce relaxation in the smooth muscles contracted of the gastrointestinal, myometrium and renal system [9]. However, detailed information regarding how the effects of hyoscine and its effects on the uterine spasm and constriction are not clear. In previous studies, only three cases were reported that treated with hyoscine before embryo transfer and there is no a comprehensive study to examine the effects of this drug on the embryo transfer [8]. Therefore this study examines the effects of hyoscine on pregnancy outcomes and its purpose is to answer the question of whether hyoscine is able to reduce uterine contractions during embryo transfer, due to its antispasmodic effect. Also, in this study, the factors affecting the outcome of ART such as age, quality and number of embryos transferred and endometrial thickness were examined.

\section{METHOD AND MATERIAL}

\subsection{Drug and Media}

Hyosine bromide was purchased from EXIR Laboratories (EXIR, Iran). All medium which related to the ART were obtained from SAGE (USA). Controlled ovarian hyper stimulation was achieved using follicle stimulating hormone; FSH (Follistim ${ }^{\circledR}$, Organon Pharmaceuticals, Inc.)

\subsection{Study Design}

The study was a randomized controlled clinical trial. Through the period from July 2012 to July 2013, healthy subjects between the ages of 21 and 41 years undergoing in vitro maturation (IVF) cycles or intracytoplasmic sperm injection (ICSI) cycles $(n=142)$ for the treatment of male factor infertility, tubal infertility, ovulatory dysfunction (ovulation complaints, endometriosis) and idiopathic infertility based on the statistical methods were randomly divided equally in two groups $(n=71)$ at the start of the cycle. Group 1 (control, $n=71$ ) without received hyosine bromide and group 2 (experiment, $\mathrm{n}=71$ ) received hyosine bromide $(25 \mathrm{mg})$.

Patient assessment included demographic information as well as medical and gynaecologic histories with physical examination and routine laboratory screening (including BMI, CBC, pap smear, TSH, PRL and viral serology). The partner underwent semen analysis, $\mathrm{CBC}$ and viral serology.

\subsection{Ovarian Hyper Stimulation}

Controlled ovarian hyper stimulation was achieved using FSH. Ovarian response was monitored by ultrasound. When two or more follicles were $\geq 16 \mathrm{~mm}$ rhCG by dose of $250 \mu \mathrm{g}$ or $500 \mu \mathrm{g}$ was used to induce ovulation.

\subsection{Sperm Separation}

Semen specimens were prepared using the swim up method and a volume of $0.3 \mathrm{~mL}$ was carried out at $36-38$ hours after rhCG injection.

\subsection{Ovarian Puncture for in Vitro Maturation and in Vitro Fertilization (IVF)}

On day 0 , at 36 - 38 hours after rhCG injection, cumulusoocyte complexes (COC) were retrieved by transvaginal ultrasound-guided needle aspiration using a prewarmed syringe. COC collection and recognition were performed by using a stereomicroscope equipped with a warm stage set $\left(37^{\circ} \mathrm{C}\right)$. Once recognized, COC were collected and then rinsed several times and incubated in the petridish containing prewarmed fertilization medium. The selected COC were inseminated with prepared spermatozoa or after removing granulosa cells surrounding oocytes were injected with prepared spermatozoa (ICSI) for the fertilization step and stored at $37^{\circ} \mathrm{C}$ under $5.8 \% \mathrm{CO}_{2}$.

Oocytes were checked for maturity and evidence of normal fertilization (two pronuclear). Fertilized oocytes were rinsed again and transferred into the new petridish containing prewarmed cleavage medium. At 48 or $72 \mathrm{~h}$ after insemination or ICSI (day 2 or 3 ), embryo development (cell number and degree of fragmentation) was recorded.

\subsection{Embryo Transfer}

In general, two or a maximum of three embryos were transferred, while excess embryos were cryopreserved in liquid nitrogen. In experimental group, embryos in about 30 minutes before injection of hyoscine $(25 \mathrm{mg})$ were transferred. After that, the patients were asked about pain. Pain with pain ruler (Visual Analogue Scale) was recorded.

\subsection{End Points}

More than two weeks (16 days) after rhCG injection, bhCG pregnancy test to determine pregnancy outcome in both groups was done and the outcome results, along with other information were recorded separately. The end point of the study was a comparison of the pregnancy rates between the two groups. 


\subsection{Statistic}

The data was analyzed by Mann-Whitney test (MannWhitney) and chi-square (Chi-square) using the software SPSS 16. Significance level of $P<0.05$ was considered.

\section{RESULTS}

A total of 142 cycles were randomized and available for investigation. None of the cases rejected to contribute to the present study. All the experimental patients were given I.M. with $25 \mathrm{mg}$ of hyosine bromide. No significant undesirable reactions at the place of injection were noted in any of the patients.

The mean ages of all of the cases was $29.8 \pm 5.1$ years with a range of 21 to 42 years. The median duration of infertility of women was $8.7 \pm 8.0$ years with a variety of 1.8 years to 14 years. The mean body mass index (BMI) was $28 \pm 5 \mathrm{~kg} / \mathrm{m}^{2}$ with a range of 21 to $46 \mathrm{~kg} / \mathrm{m}^{2}$.

Baseline characteristics were comparable in the two groups. The two groups were found to be identical with respect to age, BMI, kind of infertility, duration of infertility, number of previous trial, duration of stimulation, the type of procedures used, the total dose of gonadotropin injected, number of retrieved follicles and semen
analysis(TMC, Motility and morphology of sperm) (Table 1).

When analyzing per cycle, the overall clinical pregnancy rates were $12.7 \%$ and $29.6 \%$ for control and treated groups, respectively (Table 1). There was significant difference in the order of the clinical pregnancy between these two groups $(P<0.014)$.

Since the mean number of follicles, embryos, quality of embryos, ages patients and also duration of infertility may be have a direct relation with reproductive outcome through triggering of oocyte maturation, in vitro fertilization and embryo transfer by the given or none given hyosine, the differences between each of them were examined.

When all of the cases $(n=142)$ were classified by the mean number of oocyte into $\leq 10$ oocytes, there was significant difference in the pregnancy rates between the groups control and experimental $(28.3 \%$ vs $9.8 \%$ respectively, $P<0.017$ ). However, this difference was not strong statistically and did not affect the pregnancy rates when the control and experimental cases were categorized by the mean number of oocyte into $>10$ oocytes (Table 2).

Table 1. Baseline characteristics women who admitted to the department were comparable in the two groups.

\begin{tabular}{|c|c|c|c|}
\hline \multicolumn{4}{|c|}{ Groups } \\
\hline Baseline characteristics & Control (mean \pm S.E.) & Experiment (mean \pm S.E.) & $P$ value \\
\hline Age (years) & $26.8 \pm 2.5$ & $29.7 \pm 4$ & 0.775 \\
\hline BMI $\left(\mathrm{kg} / \mathrm{m}^{2}\right)$ & $26.9 \pm 6$ & $28.9 \pm 5$ & 0.659 \\
\hline Kind of sterility (Primary) & $36(50.7 \%)$ & $45(62.5 \%)$ & 0.87 \\
\hline Kind of sterility (Secondary) & $5(49.3 \%)$ & $26(37.5 \%)$ & 0.94 \\
\hline Duration of infertility & $4.5 \pm 5.7$ & $6.4 \pm 5.9$ & 0.09 \\
\hline Number of follicles & $5.5 \pm 1$ & $3.8 \pm 0.4$ & 0.069 \\
\hline Total sperm count & $13,500,000 \pm 906$ & $14,200,000 \pm 923$ & 0.445 \\
\hline Morphology & $9.9 \pm 3.7$ & $9.4 \pm 5.1$ & 0.965 \\
\hline Motility & $42.50 \%$ & $44.70 \%$ & 0.865 \\
\hline Follicle (Size $\geq 16 \mathrm{~mm})$ & $8 \pm 2$ & $10 \pm 4$ & 0.287 \\
\hline Pregnancy rate & $9(12.7 \%)$ & $21(29.6 \%)$ & 0.014 \\
\hline
\end{tabular}

Table 2. The variety of all of the cases $(n=71)$ were categorized by the mean number of oocytes.

\begin{tabular}{cccc}
\hline & Groups & \\
\hline & $>10$ oocyte $(\%)$ & $\leq 10$ oocyte $(\%)$ & $P$ alue \\
Control & $20(28.2)$ & $51(71.8)$ & 0.001 \\
Pregnancy rates (C) & $4(20)^{\mathrm{a}}$ & $5(9.8)^{\mathrm{a}}$ & 0.001 \\
Experiment & $18(25.4)$ & $53(74.6)$ & 0.001 \\
Pregnancy rates (E) & $6(33.3)^{\mathrm{b}}$ & $15(28.3)^{\mathrm{b}}$ & 0.001 \\
$P$ value $^{\mathrm{a} \& \mathrm{~b}}$ & $0.46^{\mathrm{a \& b}}$ & $0.017^{\mathrm{a} b \mathrm{~b}}$ & \\
\hline
\end{tabular}


When all of the cases $(n=142)$ were categorized by the mean number of embryo into $\leq 5$ embryos, there was significant difference in the pregnancy rates between the groups control and experimental $(29.9 \%$ vs $12.1 \%$ respectively, $P<0.01$ ). However, this difference was not strong statistically and did not affect the pregnancy rates when the control and experimental cases were categorized by the mean number of embryo into $>5$ embryos (Table 3).

When all of the women were stratified according to the age $\leq 35$ years, there was significant difference in the pregnancy rates of the between the groups control and experimental ( $31.6 \%$ vs $9.5 \%$ respectively $P<0.009$ ).

However, this difference was not strong statistically and did not affect the pregnancy rates when the control and experimental cases were categorized by the mean age into $>35$ years (Table 4 ).

When all of the positive pregnancy rates were stratified according to the quality grade A, there was significant difference in the pregnancy rates between the groups control and experimental $(91.3 \%$ vs $54.5 \%$ respectively, $P<0.024)$. However, this difference was not strong statistically and did not affect the pregnancy rates when the control and experimental cases were categorized by the quality grade B and C (Table 5).

When all of the patients were stratified according to their duration of infertility (I.D $\leq 5$ years and I.D $>5$ years) and endometrial thick, there was significant difference in the pregnancy rates between the groups control and experimental.

Table 3. The variety of all of the cases $(n=71)$ were categorized by the mean number of embryos.

\begin{tabular}{|c|c|c|c|}
\hline \multicolumn{4}{|c|}{ Groups } \\
\hline & $>5$ embryo (\%) & $\leq 5$ embryo $(\%)$ & $P$ value \\
\hline Control & $5(7)$ & $66(97)$ & 0.001 \\
\hline Pregnancy rates (C) & $1(20)^{\mathrm{a}}$ & $8(12.1)^{\mathrm{a}}$ & 0.001 \\
\hline Experimental & $4(5.6)$ & $67(94.4)$ & 0.001 \\
\hline Pregnancy rates (E) & $1(25)^{\mathrm{b}}$ & $20(29.9)^{\mathrm{b}}$ & 0.001 \\
\hline$P$ value $\mathrm{e}^{\mathrm{a} \& \mathrm{~b}}$ & $0.99^{\mathrm{a} \& b}$ & $0.01^{\mathrm{a} \& b}$ & \\
\hline
\end{tabular}

Table 4. The variety of all of the cases $(n=71)$ were categorized by the mean age.

\begin{tabular}{|c|c|c|c|}
\hline \multicolumn{4}{|c|}{ Groups } \\
\hline & $>35$ age $(\%)$ & $\leq 35$ age $(\%)$ & $P$ value \\
\hline Control & $29(40.8)$ & $42(59.2)$ & 0.001 \\
\hline Pregnancy rates $(\mathrm{C})$ & $5(17.2)^{\mathrm{a}}$ & $4(9.5)^{\mathrm{a}}$ & 0.001 \\
\hline Experimental & $14(19.7)$ & $57(80.3)$ & 0.001 \\
\hline Pregnancy rates (E) & $3(21.4)^{b}$ & $18(31.6)^{\mathrm{b}}$ & 0.001 \\
\hline$P$ value $\mathrm{e}^{\mathrm{a} \& \mathrm{~b}}$ & 0.99 & 0.009 & \\
\hline
\end{tabular}

Table 5. The variety of all of positive pregnancy rates were categorized by the mean quality of embryos.

\begin{tabular}{cccc}
\hline & & Groups & \\
\hline & A $(\%)$ & B (\%) $(\%)$ & $0(0)$ \\
Pregnancy rates (C) & $6(54.5)^{\mathrm{a}}$ & $0(0)$ & $0(0)$ \\
Pregnancy rates (E) & $21(91.3)^{\mathrm{b}}$ & $0(0)$ & \\
$P$ value $^{\mathrm{a \& b}}$ & $0.024^{\mathrm{a \& b}}$ & & \\
\hline
\end{tabular}




\section{DISCUSSION}

Physiological uterus movement helps the spermatozoa to go through into the fallopian tubes to fertilize the oocyte. After fertilization, decreased movements (contractions) during the mid-luteal phase are caused better contact between the blastocyst and endometrial layer so that the implantation probably is done better. Ultimately movements of the uterus during menstruation are increased that will help destroy the uterine lining [5]. During the transfer of the embryo into the uterus, the movement of the uterus is still high.

On the other hand, due to hormone therapy for stimulation of the ovary and growth of the uterus before embryo transfer, the hormonal balance is overcrowded and this may also be a barrier to the successful embryo transfer [6].

It has been shown that in the infertile women the uterus contract in contrast to the normal state is started from the fundus toward the uterine cervix. Indeed, the destructive actions of uterine motility are prevented from the transfer sperm and even embryo into the uterus. The origin of the uterine contractions is related to junctional zone in the myometrium layer [10,11].

Administration of the anticholinergic drugs such as hyoscine bromide may be able to reduce this destructive action [8]. It seems that due to the long-term sedative effect of hyoscine and also its effect on the memory can improve the pain that of obtained from the contractions of the uterus [12-14].

As mentioned in the present study, the women with successful pregnancies after embryo transfer in the treatment group were higher than women in the no-treated ones.

Hyoscine beneficial effect on embryo transfer transfer was listed by other studies. Kido et al. [8] reported that the hyoscine could be reduced uterine peristalsis in women with multiple failures in ART. In this study, the three women who were faced with the failure of ART cycles and had uterine peristalsis were given hyoscine. One of the patients was 30 years that had experienced 6 cycles failed IVF. After performing MRI, the movement of the uterus in the patient that was given hyoscine was reduced from the 7 per minute, after three minutes, to 4 per minute. The other patients were the woman aged 33 and 34 and had 7 and 4 cycles of failed IVF respectively. A similar procedure was performed on these patients. Finally, it was reported that using of anticholinergic drugs such as hyoscine may reduce uterine contractions that in turn resulted to reduce the stress on the embryo and may increase the likelihood of success of the transfer process [8].

Nakai et al. [7] investigated the effects of anticholinergic agents (hyoscine) on uterine movements by MRI techniques as well. The results showed that the mean motion of the waves in the uterine was reduced from 4.57 movements per minute before injection to 3.52 per minute. Furthermore, the severities (grade) of the uterine and also intestine contractions after administration were significantly decreased as compared with before administration [7]. Sohrabvand et al. [11] showed that the use of hyoscine can more increase the success rate of ART than indomethacin.

Other compounds can also decrease uterine contractions prior to embryo transfer. Pierzynski et al. [12] showed that administration of oxytocin antagonist decreases the uterine contractions that cause retention of embryos in the uterus. NSAID compounds by inhibiting the enzyme cyclooxygenase and subsequent preventing the production prostaglandin, is caused to reduce the contraction [15]. Fanchin et al. [5] have suggested that factors such as progesterone, $\beta$ mimetics, anti-prostaglandins and nitric oxide can reduce uterus movements. In our study, the relationship between variables such as age, duration of infertility, endometrial thickness and number of oocytes with the outcome of ART was examined. Our results showed that age, number of oocytes and embryos are associated with the ART. In our study a significant positive correlation with age less than 35 years and ART results were observed. Most cases of unexplained infertility are undoubtedly due to the natural decline in fertility with increasing age. In previous studies strongly from age, as a significant factor in the pregnancy rate, was mentioned [3,12,16-18]. Embryos derived from the older women are having a high-risk abortion because of high aneuploidy and aneuploidy occurs [12]. Also, the spindle formation in mitotic division and choromosome migration in oocyte of old women are done with trouble [4].

In our study, less than 10 oocytes were correlated with the ART result. Mcavey et al. [18] showed that the numbers of live births per cycle for 6 or more than 6 increased in compared to 5 or less than the number of cycles. In addition, it was observed that an increase of more than 10 or higher than 15 oocytes would not useful.

In our study, the fertility rate was associated with the number less than 5 embryos. Hu et al. [19] stated that increasing the number of embryos transferred in women with less than 36 years has no effect on pregnancy rate but may be increased the number of implantation. In deed in women between $36-39$ years the pregnancy rate is increased with increasing number of transfer embryos, but the patient should be given embryos with good quality. Transferring 5 embryos in women more than 40 years, regardless of the quality of embryo, could be caused pregnancy without multiple implantations unless embryos with good quality are transferred [18]. Rhodes et al. [14] found no association between embryos and pregnancy outcomes.

In our study, it was shown that there is not significant 
correlation between endometrial thickness and outcome of ART. It is stated that the endometrial thickness less than $6 \mathrm{~mm}$ can be reduced pregnancy rates. Albeit, the proper thickness of the endometrium, which is suitable for replacement, are not agreed between researchers [16]. Dickey et al. [20] showed that the $9 \leq \mathrm{mm}$ endometrial thickness at the time of HCG administration is suitable for implantation. While Lesny et al. [9] and Schild et al. [21] did not suggest the endometrial thickness as a major risk factor for embryo transfer. Rhodes et al. [14] found no correlation between endometrial thickness and pregnancy rate come, and this factor is a poor predictor of the success of the ART outcome.

The quality embryo transfer as an important factor in this study was observed. Munné et al. [22] stated that poor quality embryos which are dysmorphic and lowspeed evolution of mosaicism in compared to normal embryos have higher mosaicism, diploidy and aneupoidy. It can be declared that the embryos with poor morphology with choromosome disorders, metabolic and mitochondrial disorders are caused lower pregnancy rates [2]. Munné et al. [22] suggested that the genetic testing of embryos before transfer can be helpful in identifying these disorders. In previous studies, the association between duration of infertility and ART history has not been considered in determining the success of ART. It is also clear from our study that these parameters do not affect on the outcome of ART. Nikbakht et al. [23] have reported that cycle parameters, such as clinical pregnancy and implantation were not related significantly with the infertility duration.

Within the considerations of this prospective randomized experiment it can conclude that $25 \mathrm{mg}$ of hyosine bromide is suitable and safe treatment to reduce uterine contractions and then increase cumulative outcome of reproductive.

\section{CONCLUSION}

The clinical advantage could be demonstrated for the 25 $\mathrm{mg}$ of hyosine bromide to reduce uterine contractions and then increase cumulative outcome of reproductive. However, well-designed, further studies are essential to say a final conclusion.

\section{ACKNOWLEDGEMENTS}

The authors wish to acknowledge the efforts of Fertility, Infertility and Perinatology Research Center for its support.

\section{REFERENCES}

[1] Moramezi, F., Barati, M., Mohammadjafari, R., Barati, S. and Hemadi, M. (2012) Effect of hysteroscopy before intra uterine insemination on fertility in infertile couples.
Pakistan Journal of Biological Sciences, 15, 942-946. doi:10.3923/pjbs.2012.942.946

[2] Speroff, L. and Fritz, M.A. (2011) Clinical gynecologic endocrinology and infertility. 8th Edition, Lippincott Williams \& Wilkins, Philadelphia, 143-148, 501-518, 11501151

[3] Hourvitz, A. (2006) Role of embryo quality in predicting early pregnancy loss following assisted reproductive technology. Reproductive BioMedicine Online, 13, $504-$ 509. doi:10.1016/S1472-6483(10)60637-2

[4] Bahçeci, M. and Ulug, U. (2005) Does underlying infertility aetiology impact on first trimester miscarriage rate following ICSI? A preliminary report from 1244 singleton gestations. Human Reproduction, 20, 717-721. doi:10.1093/humrep/deh681

[5] Fanchin, R. and Ayoubi, J.M. (2009) Uterine dynamics: Impact on the human reproduction process. Reproductive BioMedicine Online, 18, 57-62. doi:10.1016/S1472-6483(10)60450-6

[6] Morizaki, N., Morizaki, J., Hayashi, R.H. and Garfield, R.E. (1989) A functional and structural study of the innervation of the human uterus. American Journal of $\mathrm{Ob}$ stetrics \& Gynecology, 160, 218-228. doi:10.1016/0002-9378(89)90126-9

[7] Nakai, A., Togashi, K., Kosaka, K., Kido, A., Kataoka, M., Koyama, T. and Fujii, S. (2008) Do anticholinergic agents suppress uterine peristalsis and sporadic myometrial contractions at cine MR imaging? Radiology, 246, 489-497. doi:10.1148/radiol.2461062091

[8] Kido, A., Togashi, K., Hatayama, H., Nakayama, T., Yamamoto, A., Kataoka, M. and Tulandi, T. (2009) Uterine peristalsis in women with repeated ART failures: possible therapeutic effect of hyoscine bromide. Journal of $\mathrm{Ob}$ stetrics and Gynaecology Canada, 31, 732-735.

[9] Lesny, P. and Killick, S.R. (2004) The junctional zone of the uterus and its contractions. BJOG, 111, 1182-1189. doi:10.1111/j.1471-0528.2004.00350.x

[10] Marikinti, K. and Brinsden, P.R. (2005) The presence of blood in the transfer catheter negatively influences outcome at embryo transfer. Human Reproduction, 20, 20292030. doi:10.1093/humrep/deh812

[11] Sohrabvand, F., Haghollahi, F., Maasomi, M., Asgarpoor, L., Shariat, M. and Hamedani, M. (2009) The effect of administrating indomethacin or hyoscine before embryo transfer on ART outcome (a pilot study). Iranian Journal of Reproductive Medicine, 7, 169-173.

[12] Pierzynski, P., Reinheimer, T.M. and Kuczynski, W. (2007) Oxytocin antagonists may improve infertility treatment. Fertility and Sterility, 88, 213.e19-213.e22.

[13] Grimes, D.A., Hubacher, D., Lopez, L.M. and Schulz, K.F. (2006) Nonsteroidal anti-inflammatory drugs for heavy bleeding or pain associated with intrauterine-device use. Cochrane Database of Systematic Reviews, 18 , CD006034.

[14] Rhodes, T.L., McCoy, T.P., Higdon, H.L. and Boone, W.R. (2005) Factors affecting assisted reproductive technology (ART) pregnancy rates: A multivariate analysis. Journal of Assisted Reproduction and Genetics, 22, 335- 
346. doi:10.1007/s10815-005-6794-1

[15] Warburton, D., Kline, J., Stein, Z. and Strobino, B. (1986) Cytogenetic abnormalities in spontaneous abortions of recognized conceptions. In: Porter, I.H., Hatcher, N. and Willey, A.M., Eds., Perinatal Genetics, Academic Press, New York City, 23-40.

[16] Warburton, D. (1987) Reproductive loss: How much is preventable? The New England Journal of Medicine, 316, 158. doi:10.1056/NEJM198701153160308

[17] Feldberg, D., Farhi, J., Dicker, D., Ashkenazi, J., Shelef, M. and Goldman J.A. (1990) The impact of embryo quality on pregnancy outcome in elderly women undergoing in vitro fertilization-embryo transfer (IVF-ET). Journal of in Vitro Fertilization and Embryo Transfer, 7, 257-261. doi:10.1007/BF01129530

[18] McAVey, B., Zapantis, A., Jindal, S.K., Lieman, H.J. and Polotsky, A.J. (2001) How many eggs are needed to produce an assisted reproductive technology baby: Is more always better? Fertility and Sterility, 96, 332-335.

[19] Hu, Y., Maxson, W.S., Hoffman, D.I., Ory, S.J., Eager, S., Dupre, J. and Lu, C. (1998) Maximizing pregnancy rates and limiting higher-order multiple conceptions by determining the optimal number of embryos to transfer based on quality. Fertility and Sterility, 69, 650-657.
doi:10.1016/S0015-0282(98)00024-7

[20] Dickey, R.P., Olar, T.T., Taylor, S.N., Curole, D.N. and Harrigill, K. (1993) Relationship of biochemical pregnancy to pre-ovulatory endometrial thickness and pattern in patients undergoing ovulation induction. Human Reproduction, 8, 327-330.

[21] Schild, R.L., Knobloch, C., Dorn, C., Fimmers, R., van der Ven, H. and Hansmann, M. (2001) Endometrial receptivity in an in vitro fertilization program as assessed by spiral artery blood flow, endometrial thickness, endometrial volume, and uterine artery blood flow. Fertility and Sterility, 75, 361-366. doi:10.1016/S0015-0282(00)01695-2

[22] Munné, S., Alikani, M., Tomkin, G., Grifo, J. and Cohen, J. (1995) Embryo morphology, developmental rates, and maternal age are correlated with chromosome abnormalities. Fertility and Sterility, 64, 382-391.

[23] Nikbakht, N. and Hemadi, M. (2012) Comparison of two doses of recombinant human chorionic gonadotropin (rhCG) during ovulation induction in intrauterine insemination cycles: A prospective randomized clinical trial International Journal of Pharmacology, 8, 259-264. doi:10.3923/ijp.2012.259.264 